\title{
Retos de la internacionalización universitaria para América Latina en el marco de la cooperación Sur-Sur ${ }^{(1)}$
}

\section{The challenges of university internationalization for Latin America in the framework of South-South cooperation}

ISSN 2071-8748

E-ISSN 2218-3345

(c) (1) (2)

BY NC SA

Blanca Ruth Orantes

borantes@utec.edu.sv https://ordic.org/0000-0001-6527-0528 Doctora en Investigación e Innovación Educativa en la Educación Superior, UGR, España, Maestra en Docencia Universitaria, Utec. Licenciada en Relaciones Internacionales, UES Directora de Relaciones Internacionales en la Utec.

DOI: https://doi.org/10.5377/entorno.v0i68.8448 URI: http://hdl.handle.net/11298/1128
Recibido: 14 de septiembre 2019 Aprobado: 28 de noviembre 2019

\section{Resumen}

El abordaje del tema de internacionalización universitaria, requiere de una visión integral que permita la convergencia de las funciones sustantivas de las instituciones de educación superior, como lo son la investigación, docencia y extensión. Esta premisa teórica que se plantea son los referentes de análisis, basado en el conocimiento y experiencia de los procesos nacionales, regionales e internacionales. Las reflexiones, dejan en evidencia la necesidad de un plan de integración que articule la heterogeneidad de los países e instituciones de educación superior, para construir y potenciar una relación de cooperación sur-sur más estrecha y efectiva que permita avanzar en los procesos de internacionalización regional, que permitirían una mayor identidad e independencia.

\section{Palabras clave}

Educación superior - América Latina. Cooperación universitaria - América Latina. Cooperación internacional educativa - América Latina. Educación internacional.

\section{Abstract}

The approach to the topic of college internationalization requires of a comprehensive vision that allows for the convergence of the substantial functions of the higher education institutions, such as research, teaching, and extension. The theoretical premise that arises is the referent of analysis based on the knowledge and experience of national, regional and international processes. Its reflections are evidence of the need for an integration plan to articulate the heterogeneity of the higher education institutions and countries, as to build and foster a closer and more effective South-South cooperation relationship that allows for an advance in the regional internationalization processes that would permit a greater independence and identity.

\section{Keywords}

Higher education - Latin America. College cooperation - Latin America. Educational international cooperation Latin America. International education.

(1) El título de este artículo nace como una propuesta en el marco del Seminario Internacionalización impartido por RIESEL, 2016, en el que participaron representantes de universidades de América Latina. 


\section{La internacionalización de la educación superior}

La internacionalización universitaria es un proceso interno y externo, que requiere de una política institucional alineada con las políticas del país y de la región. El proceso interno requiere trabajar en una cultura de internacionalización desde la base hasta los más altos niveles y viceversa, así como proveer apoyo financiero a los planes estratégicos específicos. El proceso externo, implica estar preparados con algunas herramientas, como currículum flexible en el caso de la movilidad de créditos y elasticidad en los tiempos concedidos al personal académico que incluya a investigadores y administrativos. Con ello se estaría fortaleciendo las capacidades de talento humano con mayores competencias globales, sensibilidad social y compromiso con la transformación del entorno regional.

En la experiencia que se ha tenido en la región de América Latina, la internacionalización en muchos países e instituciones no ha sido alineada ni planificada con base en intereses propios, sino foráneos. Aunque hay que reconocer que la cooperación internacional de los países más desarrollados y mejor organizados ha permitido ejecutar proyectos en los cuales, en algunos casos, no hay que poner fondos de contrapartida, pues países y sociedades como las nuestras se caracterizan precisamente por la escases económica y poca inversión en programas de internacionalización. Por ello se tipifica a los procesos de este tipo como reactivos, lo que nos hace reflexionar sobre qué se busca para nuestras sociedades del conocimiento y para la sociedad en general.

Se deben, por una parte, uniformar los criterios de la internacionalización a la luz de las tres funciones principales de la educación superior: investigación científica, docencia y extensión o proyección social.Y por otra, armonizary articular a escala regional los indicadores de internacionalización.

Una de las tensiones más visibles es la excesiva competitividad nacional entre las universidades, que las hace actuar como islas, celosas unas de otras, no permitiéndoles integrarse mediante sinergias. Esto es un obstáculo a la internacionalización vista desde cualquier perspectiva, puesto que externamente nos ven como país y hasta como región. En el caso de El Salvador, se suman algunas universidades que no han acreditado la calidad bajo los estándares nacionales, pero que aun así ya están en proceso de internacionalización sin contar en su mayoría con oficinas especializadas en el tema de gestión de la cooperación. Otra situación es la falta de una integración sinérgica regional entre las universidades que sea efectiva. Por ejemplo, hay menos movilidad académica y actividad investigadora-producción científica conjunta entre las universidades de Centroamérica.

En el tema de la investigación, se ha dado un marco de supremacía de investigadores; se difunden en publicaciones conjuntas en las que el principal investigador es de los países del primer mundo. A su vez, se ve como una oportunidad, pues en caso contrario se tendría un bajo número de publicaciones científicas de impacto, particularmente en los países menos desarrollados, en donde, además, existe un sinfín de problemas que no permiten avanzar. Y es que, en las instituciones de educación superior, a escala nacional, existen diferentes modelos: una que se dedica a aumentar las publicaciones científicas (más en Ciencias Sociales) para visibilizar la producción del conocimiento, y otra, que investiga y propone propuestas de solución o interviene, es decir, una utilidad social que se debe medir. Lo cierto que ambas deberían desarrollarse, independientemente del modelo, pues la comunidad científica y académica también necesita nutrirse de los avances de la ciencia; y eso solo es posible mediante publicaciones arbitradas, no solo con informes técnicos.

La cooperación internacional, cada vez más apoya proyectos de investigación con un resultado que mida el impacto. Nuestras sociedades necesitan transformarse y dar saltos cuantitativos y cualitativos, que permitan la innovación social y tecnológica desde lo endógeno, aprovechando a investigadores que están formados y potenciar a las nuevas generaciones, además de dar pasos importantes en la investigación experimental y en proyectos de innovación tecnológica, que es donde se observa la oportunidad de aumentar la cooperación Sur-Sur, bajo liderazgo de países de avanzada (Araya, 2015; Mendonça-Silva, 2014).

En cuanto a la extensión, esa venta de servicios a terceros está presente en muchas oficinas de spin off. La participación de los investigadores les permite mayores ingresos. Lo que significa también significa también ingresos y visibilidad para las universidades, por lo que muchas apoyan este tipo de actividad de transferencia (Kern, 2014).

Otros proyectos tienen el objetivo de intervenir a grupos vulnerables, pero bajo el esquema de asistencialismo y no de 
intervención efectiva que genere cambios auto sostenibles en la población que se interviene. Al respecto, se debería procurar una integración solidaria y endógena, un desarrollo de la universidad para el mercado. Así llenar la necesidad de generar y transmitir conocimiento relevante para la sociedad, que transforme el entorno y se contribuya a lograr una vida digna y productiva. Lo anterior, por supuesto, está vinculado con el apoyo del Estado, en cuanto a políticas de educación (Dandrea, 2016).

Se considera que las regulaciones regionales contribuyen a homologar algunos indicadores de calidad y flexibilidad en el currículo y el quehacer en investigación y extensión universitaria, en el sentido de que, cuando se acredita, hay que cumplir con esos indicadores que obligan a hacer las cosas bajo un rumbo y una norma. La situación de tensión se da cuando hay serias diferencias entre el espacio político doméstico y las regulaciones regionales. Es decir, que existen ciertas políticas educativas que conllevan solo un control, más que una contribución a la mejora de la calidad, pero que a la vez dejan espacios que permiten que no todas las instituciones se adhieran a estos procesos, lo que hace que sigan funcionando, aunque no cumplan con los criterios mínimos de calidad. La acreditación de la calidad voluntaria debería desaparecer. Se debe dar un trato igual a las instituciones que acrediten la calidad, sean públicas o privadas, cumpliendo con las normas nacionales y regionales. Así también, primero se debería cumplir con la norma nacional en los criterios homologados y luego con las regionales y mundiales.

Otra situación que merece ser analizada es que, en las regiones, siempre hay países e instituciones más avanzados, por lo que de hecho tienen mayores recursos, y en muchas ocasiones son los que buscan la hegemonía, y por supuesto el liderazgo. La clave sería cómo lograr, aun siendo líder, apoyar a los que les falta un largo camino por recorrer, respetando la diversidad y las diferencias propias de los sistemas por país, aunque se avance en un sistema de integración regional de educación superior. Es todo un reto, pero que haría una América Latina más fuerte, con mayor capacidad de negociación y auto sostenible.

\section{Perspectivas de la internacionalización universitaria}

El número de redes internacionales ha subido como la espuma en los últimos años. Entre estas hay unas que son muy especializadas y otras que no. Sin embargo, presentan muchos beneficios y oportunidades cuando son integrales; y además se tiene como objetivo internacionalizar no solo a la universidad, sino también a sus miembros (personal académico, estudiantes, etc.) Además, este aumento tiene que ver con el modelo de internacionalización que se ha retomado en las universidades, pues se trabaja la internacionalización como eje transversal en las funciones principales y no solo como un asunto del director y del equipo de la oficina de cooperación.

En la realidad, se observa que muchas de las redes resultan ser apoyo real, pero a otras les falta una visión integradora y equidad en la toma de decisiones, así como transparencia, lo que provoca desmotivación y falta de credibilidad ante los socios, y por eso estos se retiran. Otra situación que se da es que las redes venden sus servicios a precios onerosos, donde solo pueden asistir aquellas instituciones que pueden pagarlos. No obstante, hay aspectos positivos en las redes bien conducidas: exposiciones y conferencias de resultados de investigación o experiencias en extensión y docencia mediante videoconferencias, así como buenas prácticas en programas de movilidad y cooperación internacional. Proyectos entre grupos de investigación y publicaciones conjuntas que consolidan no solo al grupo, sino también a la línea de investigación y visibilidad y las oportunidades de financiamiento para la investigación. Otro fenómeno que se da con las redes internacionales es que, cuando hay financiamiento internacional, funcionan, luego se extinguen. Evidenciando la no visión de futuro, de integración y auto sostenibilidad.

En este contexto la reflexión sería en cuanto a qué tipo de redes nos conviene más y qué líderes deberían formar parte. Una de las respuestas podría ser identificar redes afines a las funciones e intereses de la universidad que esté conformada por líderes comprometidos con la educación y desarrollo. Se trata que los líderes y las universidades de países de más avanzada rompan con el modelo antiguo, para que se dé oportunidad a las universidades de países menos favorecidos, tomando en consideración que la hegemonía también se da a escala regional.

En principio, es impresionante la valentía y el esfuerzo que se ha hecho en Suramérica; unos países más que otros. Lo cierto es que hay avances, y eso es importante. En el caso de Centroamérica, se observan aún pocos esfuerzos integradores, inclusive algunos, como el Consejo Superior Universitario Centroamericano, integran universidades 
públicas y una que otra privada, peros sin voz ni voto, lo que no permite aunar esfuerzos en la acreditación de la calidad que incluyan los indicadores de internacionalización como los que cita Mérega $(\mathrm{s} / \mathrm{r})$. Hay agencias regionales en Centroamérica que acreditan carreras; y hay muchas instituciones - a manera de ejemplo- como el Instituto Tecnológico de Costa Rica, la Universidad del Valle de Guatemala, la Universidad Rafael Landívar, de Guatemala, entre otras, que han avanzado en la acreditación de carreras, lo que a la vez les permite vincularse con universidades de países desarrollados en el tema de internacionalización. No obstante, las iniciativas son individuales, no está alineada con un proyecto político regional en Centroamérica. En el caso de Costa Rica, hay un Consejo de Rectores y otros organismos que hacen esfuerzos nacionales por alinear políticas de acreditación de la calidad desde el Estado. No obstante, para Centroamérica, la alianza de cooperación entre ellos es aún mínima. Lo que hay es una oferta educativa para algunos países, en un marco de libre mercado educativo, que lo permite.

En el caso de El Salvador, el Ministerio de Educación, Ciencia y Tecnología (Mineducyt) cuenta con la Comisión de Acreditación ( $\mathrm{CdA}$ ), que solo acredita la calidad de la institución bajo indicadores que muy tímidamente abordan la internacionalización, por lo que se debe revisar y alinear con indicadores regionales e internacionales.

Se considera que, en la medida que América Latina se integre en una política regional que incluya a la totalidad de universidades por medio de un sistema normativo y políticas apoyadas con inversión pública homogénea, pero flexible por las diferencias de las regiones y países, se estaría en el camino de aprovechar las ventajas comparativas que ya tienen algunos países en el tema de acreditación de la calidad y que incluya la movilidad de talento y el trabajo de personas que provienen de un sector educativo de calidad, aunado a ello la dinámica de los sectores productivos que absorban el capital humano que se forma.

Con respecto a la homologación de títulos, todos los países y regiones deberían reconocer títulos oficiales, aquellos programas que han sido autorizados por los ministerios de Educación a instituciones reconocidas y acreditadas o no, pero a las que se les ha autorizado que impartan el programa. Un ejemplo de reconocimiento de titulaciones es cuando nuestros graduados son seleccionados para estudiar una maestría o un doctorado en el extranjero, y presentan los títulos y notas autenticadas por la entidad nacional competente, luego apostillado, y una certificación en donde se establece el número de acuerdo ejecutivo de autorización oficial para impartir el programa, así como el número de créditos para verificar que cumplen también con los créditos exigidos en el país destino. En la Utec hemos tenido esa experiencia, algunos de nuestros graduados son contratados por empresas en el extranjero (Canadá, Japón, Polonia, Estados Unidos, etc.). Sobre este tema se debería de trabajar en un organismo regional a escala latinoamericana, retomando las buenas prácticas del Mercado Común del Sur (Mercosur). La cooperación Sur-Sur es posible extenderla a Centroamérica y Las Antillas, bajo los principios de equidad y de solidaridad para el desarrollo homogéneo de la región.

\section{Programas de internacionalización universitaria}

Se considera que, a escala de América Latina, la movilidad ha existido más en programas hacia Europa, como Erasmus Mundus y hoy Erasmus+, entre otros, que han permitido mayor número de movilidades. Si se quiere fortalecer la internacionalización universitaria regional, se deben diseñar propuestas de políticas de apoyo a los países a escala regional, para la movilidad de personal académico e investigador, así como de estudiantes, particularmente hacia aquellos países modelo, pues tienen más desarrollada la investigación y la internacionalización, como por ejemplo México, Brasil, Argentina, Colombia y Chile.

Por otra parte, la internacionalización, en casa, implica trabajar en implementar y fortalecer una cultura de internacionalización que penetre en todos los estratos académicos de la Universidad, así como sensibilizar a las autoridades y a los comités para ofrecer apoyo en los procesos de internacionalización, como la homologación, el currículo flexible, los permisos para movilidad al personal académico, así como un presupuesto propio. La internacionalización universitaria es la oportunidad de transformar a la comunidad académica, a la institución y para contribuir a que el país y la región se vuelvan más competitivos, autosuficientes y auto sostenibles.

La internacionalización de la investigación es un factor clave en el desarrollo de un país y de una institución, que se visibiliza de diferentes formas; una de ellas es la divulgación y transferencia del conocimiento por medio de la ciencia y la tecnología. En muchas ocasiones estas se ven limitadas precisamente por los paradigmas de evaluación, convertidos 
en indicadores de medición de la calidad de la investigación y del perfil del investigador, que en alguna medida incide en la aceptación de publicaciones en medios de impacto y hasta en el momento de presentar proyectos en búsqueda de financiamiento.

En El Salvador, la forma que adquiere la evaluación de la investigación está determinada por un conjunto de indicadores de tres instancias oficiales: en el Mineducyt, por medio de la Dirección Nacional de Educación Superior, en el que cada año se presenta una calificación anual que contiene información estadística, como el indicador de calidad para dar cumplimiento a la Ley de Educación Superior de la República de El Salvador (2004). Uno de los indicadores es la medición de publicaciones seriadas (inscritas en el Registro de Propiedad Intelectual y publicadas por varios medios, del año anterior; a veces se quedan fuera muchas publicaciones debido a que este es un proceso que lleva tiempo) e inscripción de patentes y diseños industriales. Al respecto, no se observa que tenga mayor peso la publicación arbitrada e indexada, tampoco los proyectos en conjunto, financiados o no, con otras universidades, organizaciones gubernamentales o no gubernamentales $u$ organismos internacionales.

En el Consejo Nacional de Ciencia y Tecnología, la evaluación es similar; pero también se incluyen publicaciones no seriadas, que deberían eliminarse, pues fomenta publicaciones que no tienen ningún valor y no evaluadas por la comunidad científica. La CdA incluye indicadores similares, dando mucho valor a la publicación arbitrada, a las patentes y al impacto de la investigación. En resumen, se incluyen indicadores cuantitativos más que cualitativos, como número de publicaciones con ISBN (International Standard Book Number, 'Número Estándar Internacional de Libros') y con ISSN (International Standard Serial Number, 'Número Internacional Normalizado de Publicaciones Seriadas') [revistas propias e internacionales], patentes y diseños industriales, entre otros. También se incluyen el número de doctores dedicados a la investigación y demás personal académico. El impacto de las investigaciones no se mide con rigor académico. Al respecto, sí hay universidades que ponen mayor énfasis en hacer investigación aplicada y menos básica, basada en la existencia de muchos problemas sociales y necesidades de innovación empresarial y tecnológica que requieren desarrollar líneas de investigación que aborden estas temáticas, por lo que la calidad no se puede medir solo por publicaciones arbitradas, sino también por la intervención y el impacto que produzcan en los sectores o problemas que se tratan; y que la cooperación internacional solicita en las propuestas y en los informes de proyectos.

La evaluación de la ciencia debería ser con indicadores nacionales e internacionales, que permitan visibilizar la ciencia y su utilidad. Los planes de internacionalización deberán incluir en sus programas la internacionalización de la investigación de forma horizontal, que incluya acciones más dinámicas que visibilicen el quehacer científico de las universidades en la región, que permitan fortalecer y actualizar las competencias para la investigación, que retomen los enfoques y paradigmas con base en necesidades de desarrollo, y no investigar sin objetivos de desarrollo; que sumen al despegue de la ciencia propia que, además de ser un aporte académico científico, aborde los problemas estructurales y propongan soluciones.

\section{Retos para la internacionalización universitaria}

Existen muchos retos, que se abordan por separado en este apartado, entre ellos el de la integración de idiomas a escala regional, las estrategias de movilidad regional de créditos, y las investigaciones conjuntas interuniversitarias y con centros de investigación entre nuestros países.

Una de las tareas pendientes a escala latinoamericana es que no hay una integración de idiomas, al menos de los dos principales: español y portugués (Dandrea, 2016). Se requiere de una política agresiva que extienda las dos lenguas, y luego extender esta política hacia el resto de países de América Latina. Esto permitiría una cooperación Sur-Sur más dinámica. La oportunidad de integración, permitiría fortalecer la identidad cultural en la región del Mercosur y abriría espacios para Centroamérica y México. Integrar cooperación e inclusión en el currículo de dos lenguas además de la propia, así como el rescate del lenguaje autóctono en cada región. Sin descuidar, por supuesto, el aprendizaje del inglés, que actualmente continúa siendo el idioma universal y una herramienta importante que abre las puertas al mundo de la cooperación.

Una propuesta de acción sería muy pertinente, principalmente para fortalecer la línea de cooperación para movilidad internacional, es ofrecer el aprendizaje del portugués y conocer los aspectos culturales y lingüísticos de otras regiones de América Latina. El Mercosur debe 
implementar un plan auto sostenible a mediano y largo plazo que permita aumentar la movilidad Sur-Sur mediante intercambio de académicos y de investigación.

Con respecto a las estrategias, se deberían identificar las que requieren un trabajo en conjunto inmediato, mediato y a largo plazo. Las acciones de integración regional, deben ser a corto plazo, así como la presentación de proyectos estratégicos compartidos, la movilidad internacional regional y la necesidad de que la internacionalización de la educación superior se integre como política pública nacional y regional, incluyendo América Latina, Centroamérica y México, entre otros. Incluir programa de idioma como el portugués, pues Brasil ha crecido mucho en educación superior y puede constituirse, junto con México, en un cooperante importante, así también Argentina, Colombia y Chile.

Esto sería un paso importante, pues la cooperación de Europa y otras regiones, en donde hay países con mayores recursos y desarrollados, disminuye cada vez más; y es necesario que a escala de América Latina se unan esfuerzos de integración. Por supuesto, hay líderes económicos y políticos, en nuestra región, que tienen la capacidad de liderar y tratar de ser más equitativos, propiciando un desarrollo de la educación superior más justo y una cooperación Sur-Sur enmarcada en el principio de legalidad - se insiste en ello-, donde las instituciones cumplan con la normativa nacional e indicadores de calidad internacional. Potenciar el desarrollo del talento, la investigación básica y aplicada para sustentar el camino al desarrollo en los países aún con problemas, que son la mayoría, así como desarrollar proyectos conjuntos que aporten soluciones regionales.

Crear un sistema normativo por región tomando en consideración las buenas prácticas en países de avanzada que han visibilizado su liderazgo, como México, Brasil, Chile, Colombia y Argentina, entre otros. Cada país debería también aportar para aumentar la colaboración académica recíproca en programas de movilidad regional en las áreas prioritarias de desarrollo. También debería de haber intercambio entre profesores e investigadores universitarios. Es necesario no dejar de lado los problemas estructurales y de división que existen entre países e instituciones de educación superior, pues la solidaridad debe ser un imperativo. No obstante, la integración tiene que distinguir a las instituciones mejor preparadas con indicadores de calidad, y regular a aquellas les falte preparación, buscando así el camino hacia la integración apoyadas, de hecho, por los Estados, que deben crear y apoyar un sistema de internacionalización de la educación superior nacional y regional.

Actualmente continúa predominando la cooperación Norte-Sur en el tema de ciencia y tecnología, manifestándose en grupos de investigación de diferentes países, movilidad de estudiantes de doctorado, investigadores que aprovechan esta cooperación para mejorar sus competencias en investigación, así como con la visibilización de su actividad científica, publicaciones conjuntas lideradas por científicos del primer mundo, entre otros. No se observa equidad en la cooperación SurSur, ni siquiera con liderazgo de países como Brasil, Chile y Colombia, y otros, en cuanto a financiación de proyectos de investigación, movilidad de científicos y estudiantes de doctorado, publicaciones conjuntas, etc. Existe la necesidad de retomar la integración regional de América Latina, en donde los países de avanzada se comprometan y consideren apoyar a los investigadores, por ejemplo, de Centroamérica, particularmente de El Salvador, Guatemala, Honduras y Nicaragua, que hacen su mayor esfuerzo en su actividad investigadora y producción científica, en muchas ocasiones con escasos recursos. Los gobiernos de estos países, deben invertir en ciencia y tecnología (un fondo de desarrollo en ciencia y tecnología, formación de doctores, definir áreas prioritarias de desarrollo) y hacer esfuerzos por buscar apoyo en la cooperación Sur-Sur; que se abran nuevos espacios para aunar esfuerzos. Si no se toman medidas a corto, mediano y largo plazo, apostando a contar con recursos propios y de cooperación, el subdesarrollo humano, social y tecnológico seguirá siendo la constante de pobreza que no nos deja avanzar.

La experiencia en El Salvador, se ha caracterizado por una baja actividad investigadora y de producción científica en general. Desde el 2010 se realizó un esfuerzo y se aprobó una Política de Desarrollo en Ciencia y Tecnología, así como una Ley de Desarrollo en Ciencia y Tecnología; se han buscado recursos para formar doctores. Existe una agenda de investigación y áreas prioritarias de investigación: seguridad alimentaria, energía, salud y medio ambiente, orientadas a las ciencias naturales. Pero en El Salvador la investigación en Ciencias Sociales sigue predominando.

Se requiere mayor inversión en investigación científica orientada al desarrollo tecnológico y buscar cooperación nacional e internacional que permita participar en proyectos 
que fortalezcan la formación para la investigación con el grado de doctor, en el fortalecimiento de laboratorios; en conformar grupos de investigación sinérgicos con países cercanos, participar activamente en congresos científicos regionales, entre otras actividades de desarrollo de capacidades.

\section{La universidad integral}

y la internacionalización regional

El referente empírico del concepto que da origen al indicador debería ser un top de calidad sobre la base de experiencia, ya construida por otros países e instituciones con excelentes resultados. Este indicador, debe construirse además como un referente internacional de la calidad, integrando aspectos nacionales y regionales. Es decir, el indicador debe responder a una pirámide que integre las funciones de la universidad integral.

Los indicadores de calidad expresan la necesidad de medir cuantitativa y cualitativamente la internacionalización fuertemente vinculada con la calidad con que se forman las nuevas generaciones. La construcción de los indicadores existentes se alimenta de aportes de estudiosos en el tema, como Sebastián (2011), que a partir de estudios enlista algunos indicadores que convierten a los procesos de internacionalización en una herramienta poderosa para la transferencia del conocimiento, formación de competencias globales y multiculturales y para el fortalecimiento institucional que se refleja a escala nacional y regional. El problema surge cuando se piensan los indicadores como una decisión política o meramente para satisfacer la demanda del mercado o solo con interés nacional, pues no incluye de forma integral las principales funciones sustantivas de la universidad: docencia, investigación o actividades de extensión. En El Salvador, mi país, el Art. 3 de la Ley de Educación Superior a la extensión se le denomina proyección social (LES, 2004).

En El Salvador, insertado geográficamente en Centroamérica, su sistema recoge muy escasamente algunos de los indicadores que mencionan los autores en las lecturas recomendadas. A manera de ejemplo, apenas se consulta a cuántos congresos internacionales asistió el personal académico. El tema de movilidad de estudiantes, aunque se da desde muchos años y ha ido en ascenso, aún no se coloca como indicador de calidad. En cuanto a la acreditación internacional, existen algunos indicadores, pero se observa mayor peso en la movilidad de estudiantes, aunque en los últimos años ya se está privilegiando el intercambio de profesores investigadores y cuerpos administrativos. La agenda de internacionalización necesita incluir indicadores que permitan evaluar, en los procesos de internacionalización, resultados que indiquen el nivel de internacionalización de la investigación mediante proyectos financiados, proyectos de spin off, asistencia a congresos internacionales que certifiquen la participación del investigador o grupo investigador, patentes producto de proyectos conjuntos con instituciones de otros países, publicaciones arbitradas e indexadas, movilidad de investigadores, personal académico, estudiantes y administrativo.

Con respecto a los estándares para medir indicadores, son impuestos por los organismos acreditadores. Siendo sus expertos asesorados provenientes de países europeos o de otras regiones con mayor experiencia (Kern, 2014). Lo que falta en América Latina, y se considera que las universidades de los países pertenecientes a países del Mercosur, y particularmente las que han recorrido mayor camino (Mendonça-Silva, 2014), pueden brindar un aporte importante en la construcción de un sistema de indicadores regional, incluyendo a Centroamérica, particularmente a los países de El Salvador, Honduras, Guatemala y Nicaragua. Para ello, la voluntad política es fundamental, pero se puede mostrar una apertura para una acreditación internacional con un sistema de indicadores que integre las tres funciones principales de las universidades, y fortalezca la cooperación Sur-Sur.

La internacionalización de la educación superior, en toda su dimensión y articulando las funciones principales de las universidades, es una herramienta importante. Ha quedado claro que hay un fortalecimiento institucional que se traduce en capacidades y mejora en la calidad, y sobre todo prepara a las nuevas generaciones de egresados para que se inserten en un mundo multicultural y globalizado, pero que también debe responder a las necesidades de desarrollo regional y local, lo cual no está aislado en ese mundo complejo y en constante evolución, pues en nuestros países se tienen empresas transnacionales, grandes corporaciones mundiales, en las que se colocan nuestros graduados. Por ello se deben alinear los procesos de internacionalización, en su diseño, en las instituciones de educación superior, al plan nación y las necesidades de desarrollo. Solo así se podrá contribuir para que aquellos países que aún no lo logran salgan del subdesarrollo. 


\section{Conclusiones}

La cooperación Sur-Sur es una alternativa viable, en donde se puede negociar interuniversidades y cobijarse en parte en un fondo de cooperación regional para dinamizar la cooperación en investigación, movilidad bilateral, fortalecimiento de redes de América Latina, que permitirían integrarnos y tener mayor capacidad de negociación y toma de decisiones, permitiendo con ello homogenizar algunos de los indicadores de calidad que incluyan la internacionalización como eje transversal. La clave es articular los intereses en pro del desarrollo, tanto humano, económico, social como político, siendo la educación superior un factor clave; su función social, precisamente, es contribuir a ese desarrollo desde sus funciones principales: docencia, investigación y proyección social o extensión.

Los programas de movilidad son una oportunidad y a la vez un desafío en todo sentido: académico, cultural, en la práctica de la interculturalidad y en el aprendizaje de otros idiomas. Desde que se firman convenios multilaterales o bilaterales para movilidad, se debe contar con un plan de movilidad flexible, que pueda adaptarse a cada país y región de procedencia de los estudiantes visitantes. La estrategia y las acciones de apoyo inician desde que el aspirante se acerca para consultar y solicitar información de país, regional, y de las instituciones respectivas. A manera de ejemplo, en la Utec les compartimos información sobre costumbres, tradición, turismo, visado, etc.

Además, dar apoyo en los acuerdos académicos para que los estudiantes de todo nivel que realicen movilidad o salgan a estudiar al extranjero, tengan certeza de equivalencia de créditos académicos. Luego, cuando la movilidad ha sido aceptada, inicia otra fase que también se trabaja entre las instituciones involucradas; principalmente, las oficinas de relaciones internacionales deben dar orientación. Se provee una guía explicativa de transporte público, riesgos que existen, vacaciones y otros aspectos, para que el estudiante beneficiario se sienta cómodo y acompañado. Se presenta al grupo al que pertenecerá durante su estancia académica. No olvidando que se discute el contrato académico y la normativa interna y del país, que se debe respetar. La oficina de Relaciones Internacionales, se convierte en referente institucional para cualquier situación, prevista o no, que se presente.

Los desafíos que se presentan son que aún falta trabajar mucho a nivel interno de la institución para fortalecer una cultura de internacionalización (sensibilización); y la formación de competencias interculturales para las comunidades académicas, que permitan aceptar y respetar algunas diferencias culturales, paso importante para la armonía social.

Las actividades que se pueden implementar son: realizar jornadas de encuentros de becarios con la comunidad académica, participación en eventos culturales, aumentar el intercambio del profesorado y personal administrativo, así como giras académicas, para que vivan la experiencia. Otra estrategia es hacer videos motivacionales que muestren al país e institución origen y explicar el apoyo que tienen los grupos en movilidad. Siendo la movilidad y el intercambio una oportunidad, se debe aceptar el desafío y diseñar estrategias de promoción y apoyo antes, durante y después de finalizar la movilidad.

Las universidades latinoamericanas grandes y bien posicionadas, siempre prefieren unirse con homólogas de su nivel; y agregarían escaso apoyo en el desarrollo de la educación superior, por medio de los procesos de internacionalización, a las universidades de la periferia. Al iniciar esa construcción de las bases en que nos ordenemos en América Latina y trabajar en un proyecto de educación de calidad para todos, con agencias serias y comprometidas con la educación y el desarrollo, se estaría dando un paso importante. Por otra parte, hay que crear un fondo común que permita apoyar la investigación y movilidad regional.

\section{Referencias}

Dandrea, F. (2016). Internacionalización, política lingüística y gestión de redes. Integración y Conocimiento, (4), 169-182.

De Mendonça Silva, V. L. (2015). Universidad e internacionalización de la ciencia y tecnología en Brasil: Las facetas del programa Ciencia sin Fronteras. En J. M. J. Araya (Comp.), Aportes para los estudios sobre internacionalización de la educación superior en América del Sur (pp. 112-135). Tandil, Argentina: Universidad Nacional del Centro de la Provincia de Buenos Aires.

Kern, A. (2014). La agenda científica y tecnológica en los regionalismos de América Latina [versión de Adobe Acrobat Reader]. Recuperado de http:// web.isanet.org/Web/ Conferences/FLACSO-ISA\%20 BuenosAires\%202014/Archive/9705df48-1e10-4e3295fe-f46fc48f5992.pdf 
Ley de Educación Superior, Decreto Legislativo No. 468, Diario Oficial No. 216, Tomo No. 365 (2004).

Mérega, J. L. (2014). Propuesta de indicadores para la evaluación de la internacionalización universitaria elaborada en la Red de Cooperación Internacional de Universidad Nacional (RedCIUN). En G. Tangelson (Comp.), Desde el sur: Miradas sobre la internacionalización (pp. 107-113). Buenos Aires, Argentina: Ediciones de la UNLa.

Oregioni, M. S. y López, M. P. (2011). Dimensiones internacionales de la universidad. El caso de la Universidad Nacional de La Plata (UNLP). Buenos Aires, Argentina: Universidad de Buenos Aires.

Oregioni, M. S. y Piñero, F. J. (2015). Redes de producción y difusión de conocimiento: Un instrumento para orientar la internacionalización de la Universidad Argentina hacia Latinoamérica. En J. M. J. Araya (Comp.), Aportes para los estudios sobre internacionalización de la educación superior en América del Sur (pp. 53-78). Tandil, Argentina: Universidad Nacional del Centro de la Provincia de Buenos Aires.

Perrotta, D. (s. f.). La internacionalización de la universidad en la agenda regional: Del MERCOSUR a la alianza del pacífico. UBA-CONICET/CLACSO. Material de clase.

Rodríguez Gómez, R. (enero, 2004). Migración de estudiantes. Un aspecto de la internacionalización de la educación superior. Campus Milenio, (66).

Sebastián Audina, J. (octubre-diciembre, 2011). Dimensiones y métrica de la internacionalización de las universidades. Universidades, LXI(51), 3-16.

Sebastián, J. (2004). Cooperación e internacionalización de las universidades. Buenos Aires, Argentina: Biblos.

Velho, L. (agosto, 2000). Redes regionales de cooperación en CyT y el MERCOSUR. Redes, 7(15), 112-130. 